\title{
Equipment Condition and Environment Monitoring System of Railway Computer Room Based on Internet of Things Technology
}

\author{
Huaxun Yang ${ }^{1, a}$, Jing Liang ${ }^{1, b}$ and Ying Huang ${ }^{3, c, *}$ \\ ${ }^{1}$ Liuzhou Railway Vocational College, Liuzhou GuangXi China

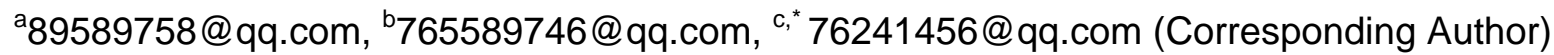

Keywords: Internet of Things Technology; Railway Room; Monitoring System

\begin{abstract}
In order to improve the efficiency of railway operation and management, and meet the needs of unmanned value development, the railway room equipment working conditions and the engine room environment are monitored with MCU and sensor technology. Administrator will be notified immediately via GSM SMS or other means in a state of emergency, which plays an important role in the safe operation and management of railway. System is divided into upper machine and lower machine part. STC12C5A60S2 single chip microcomputer is adopted as the control core, which is responsible for reading the data of each sensor. The processed data is sent to the coordinator node through the Bluetooth serial module, and then is forwarded to the remote server through GPRS module. The host computer receives the data and realizes the real-time monitoring of the environmental variables of railway room. Tests show that the system can accurately and steadily monitor the state and environment of the railway equipment room.
\end{abstract}

\section{Introduction}

The safety and reliability of the railway equipment put forward higher requirements after the rapid development of the railway industry [1]. In order to ensure the safe operation of the railway, it is necessary to build the engine room to monitor this railway equipment [2-4]. In order to accurately monitor the operation of the railway equipment in real time, a number of advanced monitoring equipment is adopted, most of these equipment are more expensive equipment, the work of the environment more stringent [2,5-7].The Railway room plays an important role in the safe operation of the railway, but also the key work of safe work [6]. However, due to some people or departments of the engine room awareness of fire safety, there are many security risks. Railway equipment failure is caused by a variety of factors, in addition to their own reasons, it may be caused by external factors, in addition, the impact of the environment on the equipment room equipment can not be ignored, such as the temperature is too high, too much humidity [4, 6-7]. It is very necessary to develop the railway equipment room and environmental inspection system in order to realize the real-time monitoring of the state and environment of the railway equipment room. Therefore, it is necessary to design a set of room environment monitoring system to monitor the working condition of equipment in the equipment room and the environment of the engine room.

There are many forms of remote monitoring. They are radio station, internet connection and GPRS transmission. In order to improve the stability and reliability of the system, single-chip technology, Zigbee technology and GPRS technology are used in the monitoring system for railway room environment, or railway signal Environment, mechanical room environment and other parameters. The current part of the technology is relatively mature which can be a direct reference to applications $[2,5,7]$.

\section{Hardware Design}

The hardware system consists of the sensor node, the coordinator node and the host computer. The decentralized distributed structure is selected to ensure that the abnormal operation of the whole system does not affect the individual sensor nodes. Hardware design includes SCM module, voltage and current acquisition module, flame sensor module, smoke sensor module, temperature and 
humidity sensor module, the human body sensing module, GPRS module and Bluetooth serial module.

STC12C5A60S2 single chip microcomputer is adopted as the control core, which is responsible for reading the data of each sensor. After processing the data, the data is sent to the coordinator node through the Bluetooth serial module. And then is forwarded to the remote server through GPRS module.

Automatic range AC voltmeter and automatic range AC ammeter which produced by Xi'an Maite Instrument Co., Ltd. are selected for collecting room equipment work voltage and current. They communicate with the MODBUS protocol with SCM, which are easy to implement.

Flame sensor circuit schematic diagram shown in Fig.1. When the flame sensor detects the flame, the resistance of the sensor becomes relatively small, and then the voltage at both ends of the sudden drop, which is lower than the negative phase input ( 6 feet) voltage, so the output voltage ofLM393 chip (7 feet) is low, but when the flame sensor does not detect the flame, the output voltage ofLM393 chip is high. Therefore, the microcontroller is used to detect the voltage status of this signal pin to determine whether a flame is generated.

Smoke MQ2 sensor circuit schematic diagram is shown in Fig.2. The working principle is similar to that of the flame sensor circuit. When the smoke sensor is detected by MQ2, the output voltage of MQ2 module changes with the concentration, the higher the concentration, the higher the voltage.
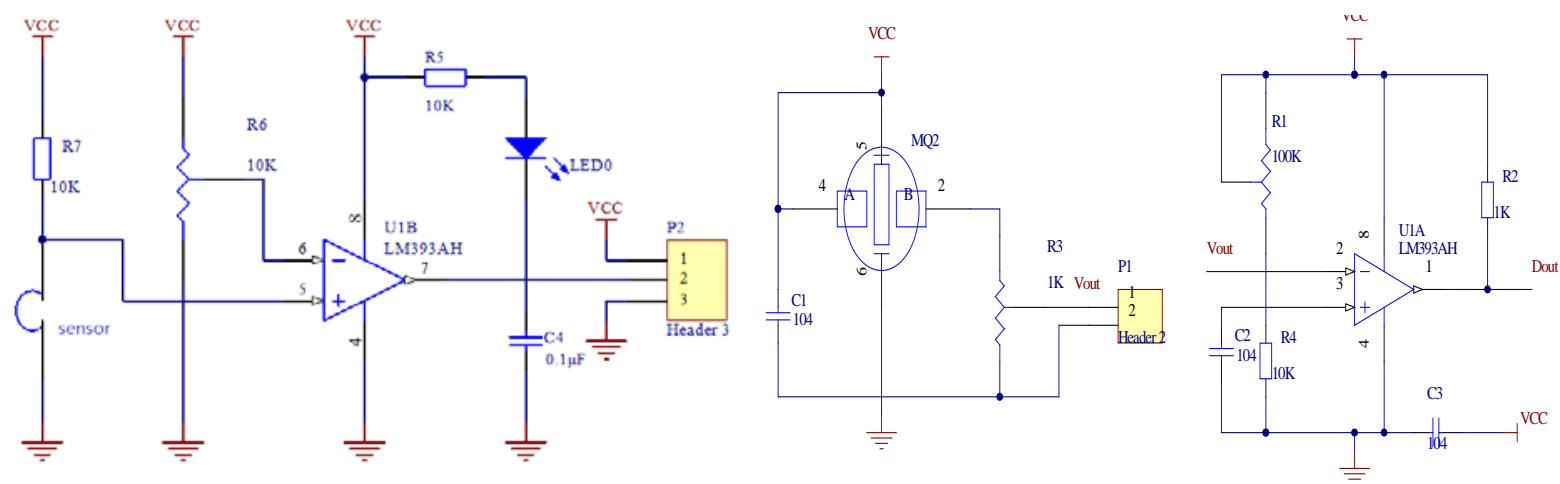

Fig.1 Flame sensor circuit schematic diagram Fig.2 Smoke MQ2 sensor circuit schematic diagram

SHT10 temperature and humidity sensors produced by the Swiss company Sensirion is used to collect the room humidity and temperature data. SHT10 is a single-chip digital temperature and humidity sensor, which has a digital output, reliable performance and stability, low power consumption and other characteristics.

SIM900A module with small size, low power consumption and wide operating temperature range is taken as GPRS module is used for communicating with SCM, using TTL level communication. Master-slave HC-05 module is used for Bluetooth serial module. Transparent transmission is adopted in HC-05 module, so the user does not need to understand any Bluetooth protocol, the use of serial communication can be directly communicated.

\section{Software Design}

STC12C5A60S2 single chip microcomputer is adopted as the control core, which is responsible for reading the data of each sensor. After processing the data, the data is sent to the coordinator node through the Bluetooth serial module. And then is forwarded to the remote server through GPRS module. The host computer receives the data and realizes the real-time monitoring of the environmental variables of railway room.

A procedure for collecting temperature and humidity is shown as following. Communication between SHT10 temperature and humidity sensor and SCM is I2C. SCM needs to start timing SHT10 sensor to read the temperature and humidity data. When SCK is high, DATA has a falling edge and remains low until SCK goes from high to low and then goes high. The DATA generates a rising edge during high level, and then the start timing of SHT10 sensor is completed. 
With the intensity of infrared light is different, far infrared flame probe to detect the signal strength is also different. When the light source is not detected, the voltage of far-infrared flame probe outputs between $0.35 \mathrm{~V}$ and $0.12 \mathrm{~V}$. While the light source is detected, the output voltage of far-infrared flame probe changes with the distance, the stronger the external infrared light, the smaller the value; the more weak the infrared light, the greater the value. The voltage is converted by the A / D converter, and then processed by the microcontroller.

The output level of the human detection module and smoke information collection module is only high or low level, which can be directly detected by SCM.

Automatic range AC voltmeter and automatic range AC ammeter which produced by Xi'an Maite Instrument Co., Ltd. are selected for collecting room equipment work voltage and current. They communicate with the MODBUS protocol with SCM, which are easy to implement. Each communication request of the MODBUS protocol must be initiated by the host. The host sends an 8-byte communication request frame in the following format, which is shown as Table 1.

Table 1 Frame Structure of Modbus Protocol

\begin{tabular}{|c|c|c|c|c|}
\hline byte1 & byte2 & byte3 & byte6 & byte7 \\
\hline Device address & $\begin{array}{l}\text { Read or write } \\
\text { command }\end{array}$ & $\begin{array}{l}\text { (Read) the starting address } \\
\text { (Write) write address }\end{array}$ & $\begin{array}{l}\text { (Read) the number of registers } \mathrm{N} \\
\text { (Write) write data }\end{array}$ & CRC check \\
\hline
\end{tabular}

The read operation succeeds and returns the data, which are processed by SCM.

The coordinator node receives the data sent by each sensor node and processes it, and then sends it through the GPRS module to the remote server. First, initialize the GPRS module and then data are sent the server after the success of the return.

Monitoring system is designed with Visual Basic 6.0, and with the following functions: Winsock communication; database data display area; data display curve; network settings function; data acquisition mode selection function; functional operation.

\section{System Test}

After the system software design and hardware design is completed, it is necessary to test the feasibility, reliability, data accuracy and stability of the test system.

Table 2 Results of Air Temperature and Humidity

\begin{tabular}{|c|c|c|c|c|c|c|}
\hline $\begin{array}{c}\text { No } \\
.\end{array}$ & Test module & Standard module(JK-8C) & error $( \pm)$ & Test module & Standard module(AS847) & error $( \pm)$ \\
\hline 1 & $20^{\circ} \mathrm{C}$ & $20.1^{\circ} \mathrm{C}$ & $0.1^{\circ} \mathrm{C}$ & $70 \% \mathrm{RH}$ & $71.5 \% \mathrm{RH}$ & $1.5 \% \mathrm{RH}$ \\
\hline 2 & $21^{\circ} \mathrm{C}$ & $21.1^{\circ} \mathrm{C}$ & $0.1^{\circ} \mathrm{C}$ & $71 \% \mathrm{RH}$ & $72.0 \% \mathrm{RH}$ & $2 \% \mathrm{RH}$ \\
\hline 3 & $21^{\circ} \mathrm{C}$ & $21.3^{\circ} \mathrm{C}$ & $0.3^{\circ} \mathrm{C}$ & $75 \% \mathrm{RH}$ & $74.6 \% \mathrm{RH}$ & $0.4 \% \mathrm{RH}$ \\
\hline 4 & $20^{\circ} \mathrm{C}$ & $21.1^{\circ} \mathrm{C}$ & $0.1^{\circ} \mathrm{C}$ & $80 \% \mathrm{RH}$ & $82 \% \mathrm{RH}$ & $2 \% \mathrm{RH}$ \\
\hline 5 & $20^{\circ} \mathrm{C}$ & $20.8^{\circ} \mathrm{C}$ & $0.8^{\circ} \mathrm{C}$ & $83 \% \mathrm{RH}$ & $83.6 \% \mathrm{RH}$ & $0.4 \% \mathrm{RH}$ \\
\hline
\end{tabular}

Table 3 Results of Intelligent Instrument Test

\begin{tabular}{|c|c|c|c|c|c|c|c|c|c|}
\hline instrument & No. & $\begin{array}{c}\text { test } \\
\text { value }\end{array}$ & $\begin{array}{c}\text { Display } \\
\text { value }\end{array}$ & $\begin{array}{l}\text { error } \\
( \pm)\end{array}$ & instrument & No. & $\begin{array}{c}\text { test } \\
\text { value }\end{array}$ & $\begin{array}{c}\text { Display } \\
\text { value }\end{array}$ & $\begin{array}{l}\text { error } \\
( \pm)\end{array}$ \\
\hline \multirow{5}{*}{ Voltage meter } & 1 & 220 & 220 & $0 \%$ & \multirow{5}{*}{ Current meter } & 1 & 1.34 & 1.33 & 0.75 \\
\hline & 2 & 220 & 220 & $0 \%$ & & 2 & 1.36 & 1.35 & $0.74 \%$ \\
\hline & 3 & 220 & 220 & $0 \%$ & & 3 & 1.31 & 1.31 & $0 \%$ \\
\hline & 4 & 223 & 223 & $0 \%$ & & 4 & 1.32 & 1.32 & $0 \%$ \\
\hline & 5 & 220 & 220 & $0 \%$ & & 5 & 1.35 & 1.35 & $0 \%$ \\
\hline
\end{tabular}

As shown in the Table 2 and Table 3, data collected from sensor nodes are correct, errors-free.

The monitoring system software also works correctly, which is shown in Fig.3.Tests show that the system can accurately and steadily monitor the state and environment of the railway equipment room. 


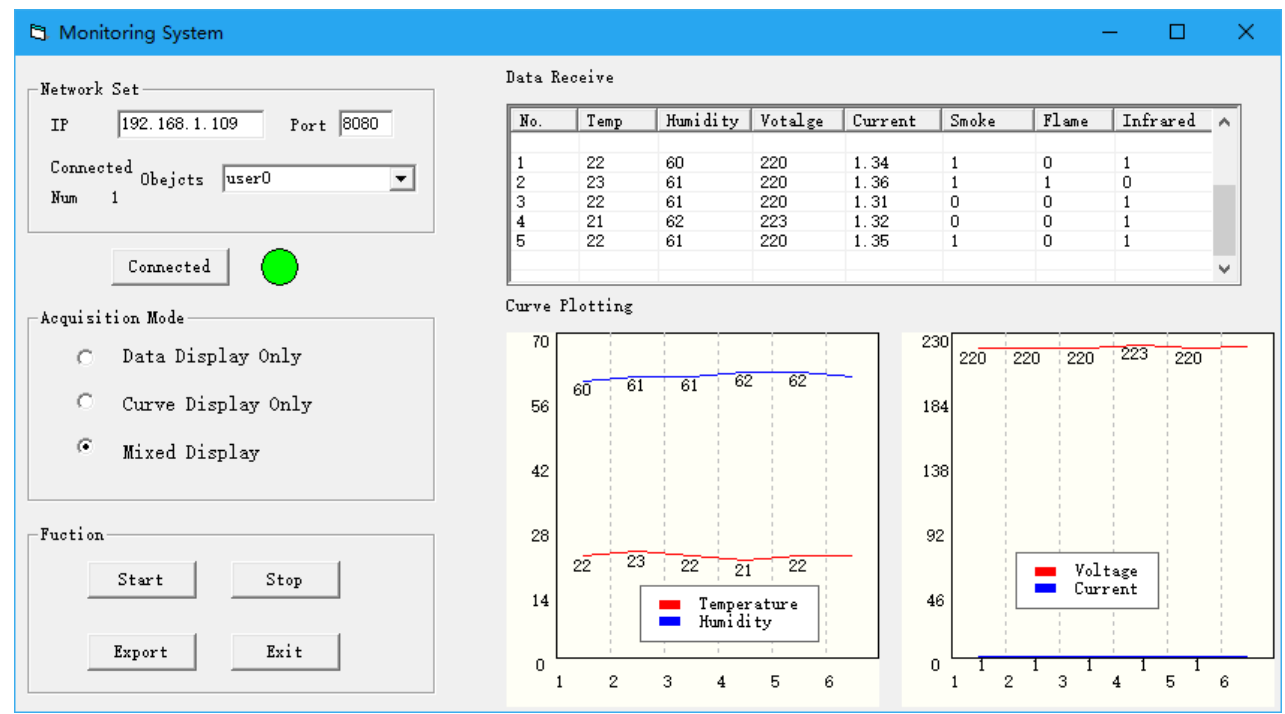

Fig.3 Operation interface diagram of monitoring system

\section{Summary}

Equipment condition and environment monitoring system of railway computer room based on internet of things technology is designed. System is divided into upper machine and lower machine part. Lower machine part design includes SCM module, voltage and current acquisition module, flame sensor module, smoke sensor module, temperature and humidity sensor module, the human body sensing module, GPRS module and Bluetooth serial module. Upper machine part design includes the PC monitoring system and mobile terminal monitoring system, which has the function for data displaying, saving, and data exporting. Tests show that the system can accurately and steadily monitor the state and environment of the railway equipment room.

\section{Acknowledgements}

This work was financially supported by the Project of Outstanding Young Teachers' Training in Higher Education Institutions of Guangxi and a Guangxi University research project.

\section{References}

[1] TIAN Xiao-Dan. Optimization of Environmental Monitoring System for Railway Communication Room [J]. Electronic Technology and Software Engineering, 2015, (05): 53.

[2] Zhao Tao. The Design and Implementation of Railway Machine-room Environment Monitoring System [D] .Guangzhou: South China University of Technology, 2011.

[3] Liu Daohuan. Research and Design of Railway Signal Equipment Room Environment Monitoring System Based on ARM9_VxWorks [D]. Nanchang: Nanchang University, 2010.

[4] Pang Bo. Research and Implementation of the Technology of Telecommunication Machine Room Remote Monitoring and Administration [D]. Beijing: Beijing Jiaotong University, 2008.

[5] Yan Hao Bo. Research and Development of Railway Signal Power Monitoring System [D]. Tianjin: Tianjin University of Technology, 2007.

[6] Li yuejie. Development and Research of Railway Signal Environmental Monitoring System Based on ARM [D]. Tianjin: Tianjin Polytechnic University, 2007.

[7] Zhou Chunmei. Environment Monitoring System of Railway Signal Machine Room [D]. Tianjin: Tianjin University of Technology, 2007. 